\title{
Designing Effective Playful Experiences for Sustainability Awareness in Schools and Makerspaces
}

\author{
Georgios Mylonas \\ Industrial Systems Institute, Athena Research and \\ Innovation Center \\ Patras, Greece \\ Computer Technology Institute \& Press "Diophantus" \\ Patras, Greece \\ mylonas@athenarc.gr \\ Andreas Friedl \\ ovos media \\ Austria \\ af@ovos.at
}

\author{
Joerg Hofstaetter \\ ovos media \\ Austria \\ jho@ovos.at
}

\begin{abstract}
Sustainability awareness in young people, and especially students, is growing in importance. To this end, serious games and playful experiences are used to enable students and teachers to actively study climate change issues and respective challenges. Many approaches have been proposed, ranging from quiz-based web applications to full-blown game experiences. We discuss several related design aspects through the lens of our own experience with gamification inside educational environments. Our results derive from the use of a playful web application focusing on sustainability and energy issues, developed within the GAIA project and used in 25 schools with 3762 registered users. We also present results from surveys answered by 723 students and 32 educators. Our findings show that a simple playful experience can yield good results within educational environments when taking into account their constraints, integrating the intervention to schools' everyday life and placing it within a strategy that includes other tools.
\end{abstract}

\section{CCS CONCEPTS}

- Applied computing $\rightarrow$ Interactive learning environments; - Human-centered computing $\rightarrow$ Empirical studies in HCI.

\section{KEYWORDS}

Gamification, Sustainability, Energy awareness, Empirical studies, Internet of Things

\section{ACM Reference Format:}

Georgios Mylonas, Joerg Hofstaetter, Andreas Friedl, and Michail Giannakos. 2021. Designing Effective Playful Experiences for Sustainability Awareness

This work is licensed under a Creative Commons

Attribution-NonCommercial-NoDerivs International 4.0 License.

FabLearn Europe / MakeEd 2021, June 2-3, 2021, St. Gallen, Switzerland

(C) 2021 Copyright held by the owner/author(s).

ACM ISBN 978-1-4503-8989-1/21/06

https://doi.org/10.1145/3466725.3466755

\author{
Michail Giannakos \\ Norwegian University of Science and Technology \\ Trondheim, Norway \\ michailg@ntnu.no
}

in Schools and Makerspaces. In FabLearn Europe / MakeEd 2021 - An InternationalConference on Computing, Design and Making in Education (FabLearnEurope / MakeEd 2021), June 2-3, 2021, St. Gallen,Switzerland. ACM, New York, NY, USA, 9 pages. https://doi.org/10.1145/3466725.3466755

\section{INTRODUCTION}

Climate change and its effects have initiated various processes in terms of understanding the issues causing it and the ways to respond to it. Although this has been ongoing for decades, such activities have only been intensifying in the last decade. The United Nations (UN) Climate Summit in Paris in 2015 was recognized as a major breakthrough in environmental governance and global policymaking [2]. Global climate change and sustainable development require novel approaches to energy consumption across a range of human activities [21] and shifts in technologies, techniques or infrastructures, meeting today's demands, without compromising the needs of the future generations [20]. To this end, there is an acute need to improve awareness on individual and societal levels, especially at the younger populations.

The research community, as an active part of society, has been looking into ways to sensitize a wide range of end-user groups towards sustainability and add new tools to help tackle this crisis $[18,28,35]$. In particular, greater attention should be paid to children's awareness, agency and sustainable behavior, by considering research on the flows of environmental knowledge between the spaces of children's lives [43]. One such path taken by the community is the use of gamification mechanisms, in order to engage children on related aspects and give them incentives to act responsibly, e.g., save energy at the school or at home [15, 22, 35, 39]. In this context, researchers have targeted a wide range of end-user groups/communities, like civil servants, office workers, public housing tenants, among others [20].

However, up until recently our societies have not been as active on this front as someone would have expected. For instance, recent research shows that people lack a basic understanding of how energy is produced and transmitted, the basic units of consumption, and the implications of many day-to-day actions [21, 29]. Despite the obvious reason for why school students are an important user-group (i.e., future citizens that need to learn the values, 
behaviour and lifestyles required for a sustainable future), the educational community is also an excellent candidate for energy saving since according to Eurostat [1] educational buildings cover $17 \%$ of total non-residential floor space in the European Union (EU). In other words, this sector has a tangible effect on the overall energy consumption and improvements on that direction will help EU to achieve its energy savings goals [4], and promote behavior change [3]. In this sense, school curriculum and informal learning activities would be an excellent starting point in order to achieve sustainability awareness and energy saving behavior, resulting in substantial long-term benefits.

Thus far, the actual implementation and integration of serious games and playful experiences related to sustainability and energy by the research community has followed a range of different paths $[20,21,35]$. On the one hand, there have been quite a few implementations that offered games of considerable size and depth, which follow the design of games like city simulators but with a sustainability and renewable energy focus [20]. On the other hand, simpler experiences have attempted to focus on educating end-users by presenting them with information in a playful manner, e.g., in the form of interactive quizzes [21]. However, those implementations are agnostic to the school-related conditions and restrictions, such as the available time for extracurricular activities, the experience and background of the educators, available resources, or even the school building. Another important element is students' age, e.g., primary school students have a different knowledge level than lower secondary students. Moreover, although the use of gamification is used intensively in related work [14, 20, 22], the use of data and analytics coming from the real world is not as common.

In this study, we focus on the following research questions (RQ):

$R Q 1$ How can simple playful interventions be effectively employed inside schools (e.g., classrooms, makerspaces)?

$R Q 2$ What kind of game mechanics, and under which conditions, can be put into practice in schools?

$R Q 3$ How can gamified interventions be successfully employed in schools?

To respond to the aforementioned RQs, this work presents the results coming from a playful intervention, called the GAIA Challenge [11]. By this, we mean an online platform that uses gamification elements, without having the depth, complexity and duration of a dedicated serious game, and which was used as a playful introduction to sustainability and energy saving. The Challenge was utilized in schools aiming for increasing sustainability awareness and achieving energy savings through behavior change, with students from primary, middle and high schools from 3 European countries (Greece, Italy and Sweden) participating in the Green Awareness In Action [6, 31] (GAIA) project. By the end of the project, a total of 3762 users had registered to the platform, allowing us to implement a large-scale study. Data regarding the students' participation and attitude change were collected via analytics and questionnaires. The results indicate that simple gamification mechanics $[9,23]$ and techniques can be very effective, while relevance (i.e., activities need to be related to the curriculum), as well as placing the intervention inside an overall strategy (i.e., part of a bigger set of activities and not a one-size-fits-all solution), aligned with schools' routines is of great importance.

\section{BACKGROUND AND RELATED WORK}

\subsection{Gamification and energy saving}

Gamification, defined as "the use of game design elements in nongame contexts" in [16], is an approach that has been gaining popularity in both the fields of sustainability and educational research. The use of gamification in education was recently discussed in [17], where the need for additional systematic studies to understand the actual effect of this approach is supported by the authors, due to the fact that most of the studies focus on short-term effects and results, and do not consider how such interventions can bring long-term effects and system changes. In this context, [30] provides a review of recent bibliography in serious games and gamified interventions for sustainability, concluding that such approaches can help foster sustainability, while there is a wealth of different approaches and deployment strategies.

Regarding the use of gamification for energy saving, the work of Johnson et al. [24] provided a recent review of the use of such techniques, while Ro et al. [36] reports on positive outcomes of gamification on both sustainability awareness and energy savings. Zhao et al. [45] studied the interplay and effect between the various technologies used inside a building for monitoring/control, concluding that both technology advances in building equipment/devices and the ways humans interact with them can lead to energy savings. Moreover, as regards the use of competition in this context, the relation between competition and sustainable behavior was studied in [40], where the authors claim that utilizing competition in actions promoting sustainability is a valid "marketing" strategy, since it appeals to both groups that are less motivated and those that are more inclined to participate in such activities. This is also discussed in [44], where both competitive and collaborative approaches are studied, and which have led to similar energy saving results. The results presented in [34] seem to verify the value of competition to a certain extent, where results from long-term energy-saving competitions among 4 schools led to modest energy savings. Our work discussed here agrees with their findings, while we also provide results on the educational dimension of the schools' participation.

Regarding the use of gamification for energy saving, a characteristic early example of the use of a serious game towards energy and sustainability awareness was Enercities [26], reporting increased awareness and more positive attitudes as regards energy saving. Opower [27] was one of the earliest commercial applications utilizing behavioral science-based mechanisms, aiming to produce energy savings in the real world by partnering with energy providers and offering consumers located in the same neighborhood targeted information. Real-world results point to $2-3 \%$ energy savings in the long term. The work of Johnson et al. [25] provided a recent review of the use of such techniques, while Ro et al. [37] reports on positive outcomes of gamification on both sustainability awareness and energy savings. A collection of sustainability-related serious games is available on Games4Sustainability [5].

Recent projects like Charged [19], Entropy [8], GreenSoul [10], Tribe [12], have pursued IoT- and game-based approaches towards increasing awareness on behavior-based energy efficiency in public buildings. However, although schools may be considered examples of public buildings, the software and hardware artefacts produced in these projects are not designed to be included in a school's 
curriculum; in fact, they target adults, making it difficult for teachers and parents to apply such materials with young students. With respect to gamification utilizing IoT in the context of sustainability, and specifically for energy and water, a recent survey on the subject is provided in [13].

\subsection{Effectiveness of gamification in sustainability and education}

Regarding the successful integration of gamification techniques for energy saving and sustainability awareness, while there is a large number of works that report on positive outcomes, e.g., [27, 31, 37], at the same time there are also works that report on less positive results and stress the acute need for taking action [41]. This controversy is partially based on the fact that there is no commonly acceptable way on how results are measured and reported; [38] provides a detailed discussion on common pitfalls and mistakes made by teams working on these issues. It concludes that there is a number of works that do not sufficiently back up their findings with adequate evidence, resulting in reports which are oftentimes disputable and provide contradicting results. For such evidence to be useful for the society and activate policy and practice, it needs to be connected with rigorous experiments and measurements. Dos Santos et al. [20] provided a comprehensive review of recent works on the use of gamification techniques for approaching learning activities related to sustainability. It provides a categorization of related serious games based on their focus, namely, educational and motivational games for sustainability. It concludes that existing results are somewhat partial and there is a need for a more rigorous and systematic approach in evaluating the effectiveness of such approaches. Another recent work reporting on the causes of the failure in practice of gamification approaches applied in an educational context is [41]. The authors present a set of "heuristics" to evaluate whether a gamification approach applied in education has a potential to be successful, such as "avoid obligatory uses", "make the system flexible" and "align gamification with the goal of the activity in question". In [42], the authors argue that a more thorough understanding of how gamification works in education and especially with respect to motivational processes and needsupporting game elements, i.e., "gamification in education that is designed to foster learners' basic psychological needs satisfaction”.

Regarding the current state of the art in inclusion of sustainability and other related aspects in the educational domain, there is a lot of activity taking place with respect to inclusion of makerspace elements in school curricula, aided by the availability of IoT hardware as well. The work in [33] summarizes recent activity within the Maker Movement approach, presenting relevant recent findings and open issues in related research. Recent projects like Entropy [8] target diverse end-user communities and do not focus specifically on the educational community, or leave it out completely.

With respect to the work presented in this paper, we follow a different approach than the vast majority of the gamification approaches studied in the bibliography; instead of focusing solely on a gamified/playful strategy, we have placed the Challenge inside a set of tools to achieve our goals. In addition, the design of the Challenge is largely compatible with strategies outlined/proposed in works like [20] and [41], especially aligning gamification with

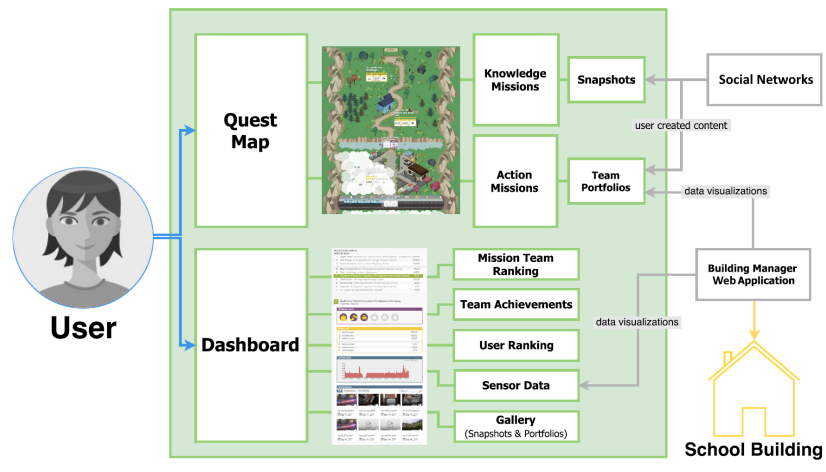

Figure 1: The overall architecture of the GAIA Challenge.

the goal of the activity in question and offering a flexible approach, offering among others, a focused and seamless experience that goes hand by hand with curriculum goals and encouraging group activity to reach learning goals, instead of rewarding specific individuals. The gameful intervention discussed here was one of the pillars used to introduce students to sustainability concepts, keep them engaged and allow them to "compete" across schools. Regarding our own previous related work, [31] provides a discussion on the overall approach and strategy of GAIA and presents some early findings as regards both energy savings and feedback from students and educators. [32] discusses positive results received via large-scale pilot activities with groups of students using an IoT-based lab kit.

\section{METHODS}

\subsection{Context and description of the challenge}

The Challenge is an online playful introduction to sustainability and energy saving, targeting mostly school students. Its architecture combines a quest map with a dashboard and uses sensor data coming from school buildings (Fig. 1). It consists of a number of missions (Fig. 2) and utilizes gamification mechanics to motivate students to engage in energy saving topics by collaboratively working on online "quests" and participating in real-life activities. Moreover, students experience their impact on the facilities' energy consumption over the course of the Challenge, while also competing and comparing against other classes and schools. Overall, it has the following goals:

- Motivate children to engage with energy saving topics.

- Offer a variety of online quests (quest map).

- Allow children to participate in real life activities (class activities).

- Introduce competition elements to children via scoreboards with data from other schools and other countries.

- Allow students to share their achievements with their peer group.

- Scaffold an in-situ experience and engage children on their school's energy consumption.

Gamification in the GAIA Challenge is utilized in the following ways:

- Students and teachers pick up tasks from the Challenge and either work on these tasks directly in the Challenge, or they 

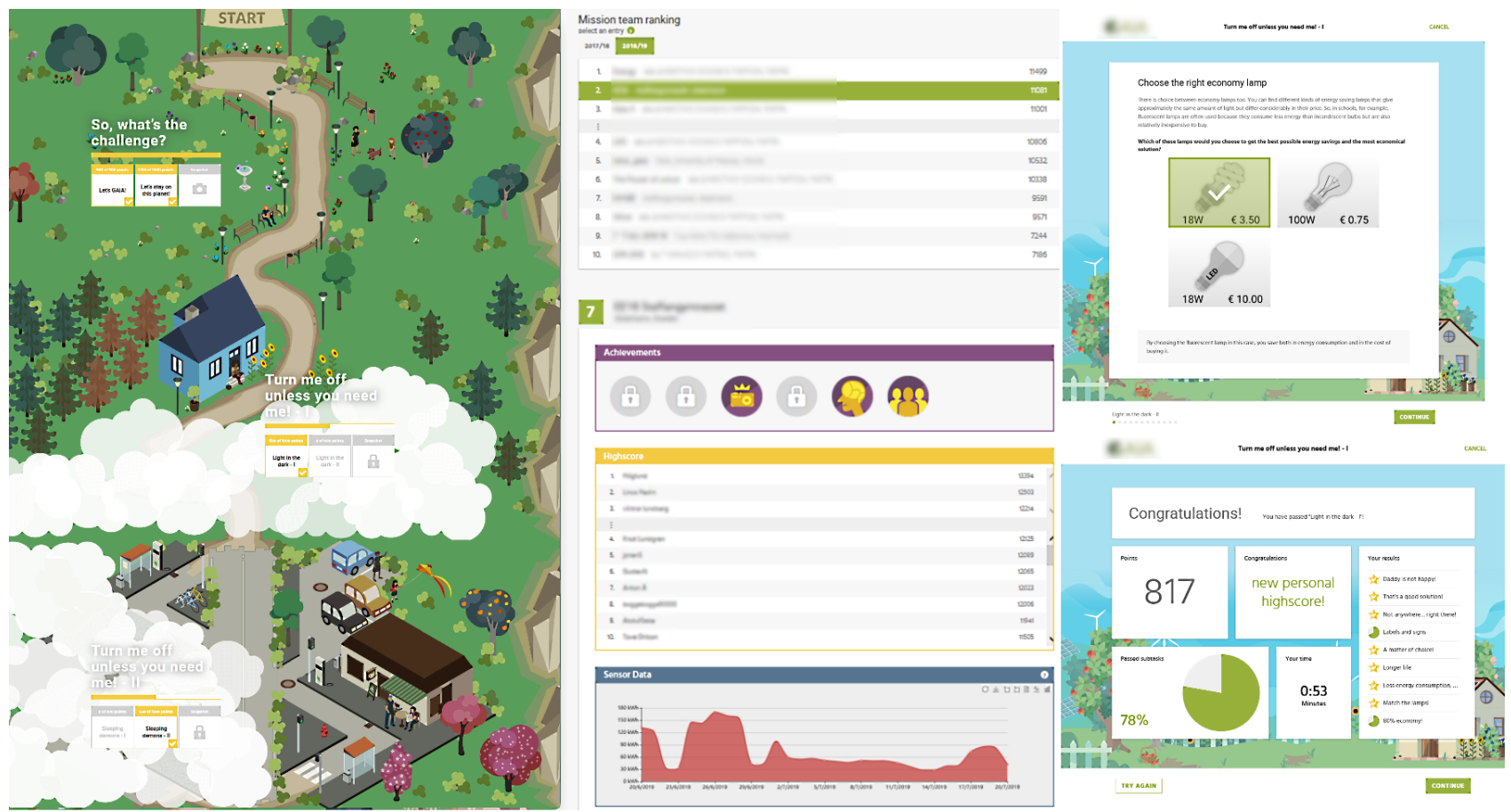

Figure 2: Examples from the Challenge: the quest "map" where players can select a "mission" (left), ranking and score tables, together with power consumption readings (center), screenshots from content inside the missions (right).

use it together with a number of other tools produced in the GAIA research project.

- Knowledge missions are played directly in the Challenge browser window, but may require additional research or actions from other GAIA applications, like a web-based building manager application.

- Findings, results and ideas can be shared in the form of "Snapshots".

- For action missions, no direct interaction within the Challenge is required, but students instead have to engage with their school community, the school building and the other applications.

- The results of Action Missions can be documented in "Portfolios", supplemented with photos/videos by students.

- Visualizations of sensor data, can be grabbed from a Building Manager App and embedded to Snapshots/Portfolios.

\subsection{Procedure}

Activities using the Challenge took place within the GAIA project across Greece, Italy and Sweden. There was an initial small-scale pilot activity, mainly for testing purposes, during 2017. The design and the majority of the content was finalized in early 2018, while large-scale pilot activities took place in school years 2017-18 and 2018-19. By the end of the project, a total of 25 schools had participated in the pilots, with most schools being active in both school years.

\subsection{Data Collection}

To evaluate the effectiveness of the intervention and investigate its benefits, we collected data via 3 different sources: i) analytics generated by students' activity during the Challenge, ii) responses to an online survey that was sent out to participating students, and iii) questionnaire responses from educators.

3.3.1 Analytics. During the study, we captured children's interactions (e.g., achieved score for each session) via web analytics. In particular, we collected events using the Matomo platform [7], in order to track traffic on the Challenge website, while we monitored the overall players' progress through the internal database of the Challenge. By design, we kept minimal data about players such as the completion rate of missions, while students could not upload avatar photos. Furthermore, registered users are auto-deleted after 10 months of inactivity together with their respective data.

3.3.2 Questionnaires. Students' Survey Close to or shortly after the end of the project activities (depending on the schedule of each school) in the school year 2018-2019, an online questionnaire was administered to participating students. The aim of this post-activity survey was to establish the impact of the project activities and experience overall in the course of the school year on students' awareness of, attitudes to, and actions for energy efficiency.

Teachers' Survey We also conducted a second, separate, survey in 2020, with 32 educators from 3 countries, who were actively involved in the implementation of in-class activities in the project, therefore, had a direct experience of the utilization of the Challenge 


\begin{tabular}{l|l}
\hline Total registered users (May 2019) & 3.762 \\
\hline Active users & 1.747 \\
\hline Auto-deleted users (deleted after an inactive period) & 2.015 \\
\hline Active teachers & 55 \\
\hline Mission teams & 165 \\
\hline Portfolios created & 38 \\
\hline Snapshots created & 788 \\
\hline
\end{tabular}

Table 1: Overall statistics for the Challenge at the end of GAIA.

in their classes and the response of the students. The questions were:

Q1 Do you think the Challenge helped you save teaching time due to the introduction to sustainability and energy saving that it offered to students?

Q2 Do you think it meets the level of knowledge of your school's students?

Q3 Have you noticed any change in your students' energy consumption behaviour after they had played the game but before the project's pilot actions took place?

\subsection{Data Analysis}

As mentioned, the collected data consists of 2 types, analytics and questionnaires. An appropriate data analysis was used for each different set of data. Per students' analytics, we used descriptive statistics to understand the students' overall engagement with the Challenge and aggregated time series visualization, in order to understand how their usage behavior changed over time. In addition, we used descriptive statistics to understand the students' overall reported experience, as demonstrated from questionnaire responses. Regarding the analytics from the Challenge website, we include here results from the whole active period of the project, i.e., between the public opening of the Challenge on Spring 2017 and May 2019. As regards the players' data from the Challenge database, we include here results from a representative "screenshot" on Spring 2018, which includes only the active players at that point, i.e., the school year 2017-18. We selected this period mainly due to having a larger user sample for that year.

\section{RESULTS}

\subsection{Results at the end of the project}

By the end of the project, 73 teams appeared to be active in the Challenge, with 7 teams achieving a score above 11.200 points (the total of regular points each user can earn). This essentially means that they must have played not just the complete learning part of the challenge, but also achieved big portions of the limited time bonus (there is a maximum of 3780 bonus points). The actual number of active users is lesser than the total number of registered users, because we have placed a mechanism for auto-deleting inactive users after a period of 6 months, i.e., a large number of students were auto-deleted. This is consistent with our ethics and privacy policy, i.e., keeping as little information about the users as possible, and deleting it after the time period which is no longer required.
Visits per local time: Our data show that main play hours (Fig. 4) were during class time (i.e., between 08:00 and 14:00), but that the Challenge is being played in the afternoon as well, and even in the evening. This could be because of a flipped classroom setting or, even better, by students continuing to play the Challenge from their homes. In all cases, it seems that the students really liked the Challenge and dedicated serious time in completing its missions.

Visits per weekday: Interestingly, visits are not limited only to school days, with $11 \%$ taking place during weekends (Fig. 6), which is another indication of the students' overall acceptance of it and their continued engagement.

Duration per visit: The average session time (Fig. 7) of all users is very high for this sort of application, and in comparison with similar gamified systems. Almost $50 \%$ of the sessions had an average duration of over 10 minutes, i.e., when a significant percentage of the students logged in, they started and completed a mission. Regarding the many shorter sessions, apart from students entering and quickly losing interest in the Challenge, one other possible explanation could be that they logged in to check the ranking of the schools.

Visits per device: $28 \%$ of the Challenge usage comes from smartphones, although it was designed for big screens. The vast majority of visits comes from desktop/laptop users, as expected, with a sizable part of visits from tablets.

\subsection{Mission completion rates for school year 2017-2018}

As mentioned, we focus on the "screenshot" of our database on May 2018. At that point, there were 1256 active users in the database (13 were researchers/test accounts). An interesting statistic was the mission completion rate for users who had started a mission at least once, which was on average $87.45 \%$ for all missions $(87.2 \%$ for Knowledge missions). It can be concluded that almost 9 out of 10 users who started a mission then completed it at least once, i.e., they found it engaging enough to complete it through. Regarding the number of missions completed by the students, Fig. 8 shows the distribution of the number of completed knowledge missions per user. Almost half of the students appear to either not complete a single mission (16\%), or got stuck/stopped at $1(17 \%)$ or $2(18 \%)$ missions. Since most players followed a linear route in gameplay, such users stopped at "level" 1 or 2 . On the opposite end, $21 \%$ of the users completed all knowledge missions, or played missions in an additional language. In the space between, percentages are between 3-6\%.

However, this picture changes when we look at the user statistics for each language in the Challenge. In general, the language used in the Challenge roughly corresponds to the country of origin of the user, although there are a few cases of users playing the Challenge in more than 1 language for some missions. Moreover, via the language used we can roughly categorize the users: for English the vast majority of the students were 17-18 years old, while for Italian the users' age was 15-16, and for Greek it ranged between 10 to 15 years old. We can see that for English there is a quite steep drop in interest after missions 1 and 2, while for Greek results are quite different with a much smoother curve. For Italian, the curve is even flatter, and after mission $4,60 \%$ continued to mission 9 . 


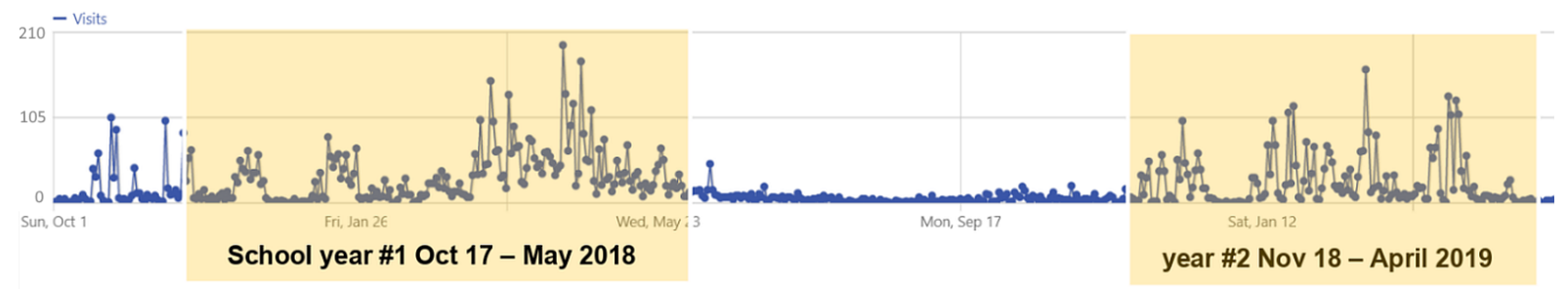

Figure 3: Visits to the Challenge website during the periods in which the pilot activities took place.

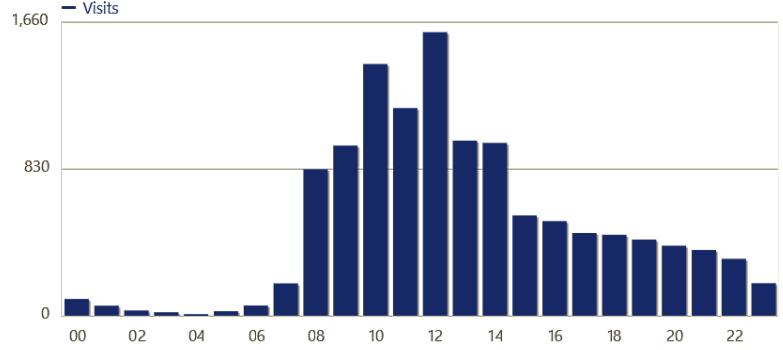

Figure 4: Visits by players to the Challenge website, by local timezone. Notice that visits persist even after 14:00, and continue until late in the afternoon and even at night.

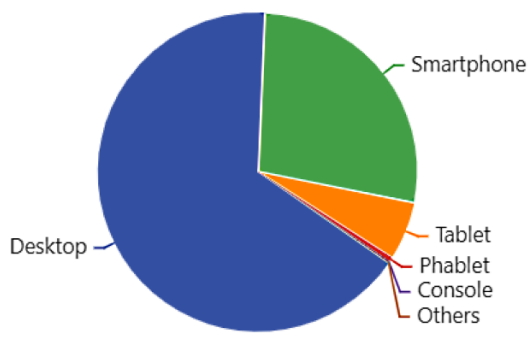

Figure 5: Visits by players to the Challenge website, by device type.

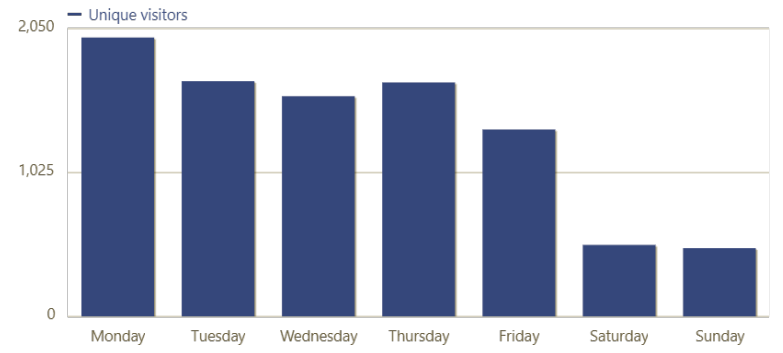

Figure 6: Visits by players to the Challenge website, by week day. Apart from the visits during the working week, we can also see that player visits continued throughout weekends as well.

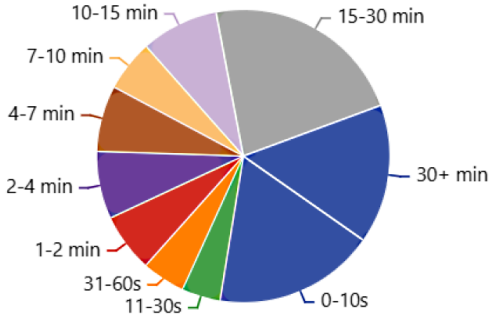

Figure 7: Distribution of the average session times of all users throughout the activities of the project. Close to $50 \%$ of the average session times are over 10 minutes.

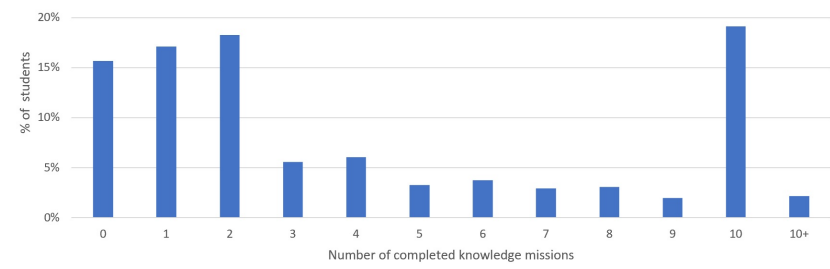

Figure 8: The overall distribution of the number of completed knowledge missions per user.

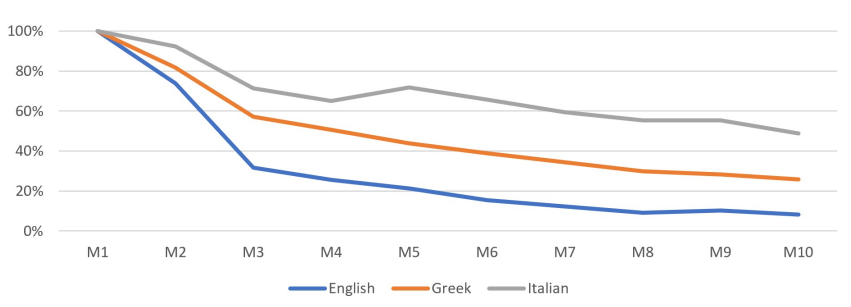

Figure 9: The distribution of the number of initiated knowledge missions per user per language.

\subsection{Mission Statistics}

From our statistics, we found that in the Challenge there have been 22.429 started tasks altogether. The breakdown into the statistics for each knowledge and action mission is included in the following two tables. As a reminder, each mission has two tasks. A player can open a task multiple times, e.g., in order to improve mission completion statistics. The tasks in Action Missions can be started only by players, granted the Action Mission has been started beforehand 
by a Teacher for the Player's Mission Team. Not many users have started/opened the contents of Action Missions in the Challenge. There could be multiple reasons for this: a) not all players were part of a Mission Team, b) not all teachers started Action Missions for their Mission Teams, c) some students might have worked on Action Missions in groups.

\subsection{Questionnaires results}

4.4.1 Students questionnaire results. Overall, 723 students participated in the questionnaire survey, which corresponded to $42.5 \%$ of the total 1702 students participating in the project's activities in that school year, thus constituting a strong representative sample of that population. The participation in the survey was balanced in terms of gender ( $49.6 \%$ female respondents), and comprehensively covered the different participating schools and regions. The responses came from both primary (45\%; up to 11-12 year-old-students) and secondary (55\%; 12-18+ year-old) education students. Through this post-activity online questionnaire, the consortium also sought to gain insight into the impact the different aspects of the project intervention had on students. On the one hand, the survey asked students to state to what extent they liked: a) the Challenge, b) to observe and use sensor data and measurements, and c) a series of lab activities using IoT.

Overall, approximately half of the students stated that that they liked these project activities "much" or "very much" (54.5\% the Challenge, $50.4 \%$ the lab activity, and $46.9 \%$ the data and measurements). The overall somewhat positive to positive response, i.e. excluding the responses 'not at all' and 'a little', approaches or reaches the level of three quarters of the respondents $(75.9 \%$ the Challenge, $73.1 \%$ the data and measurements, and $72.1 \%$ the lab activity). As becomes evident in Fig. 10, the Challenge tended to be liked the most, followed by the lab activities. In addition, students were asked to self-assess to what extent they believed they had learned something about energy (Table 2). From their answers, 57.1\% strongly believed that they had learned something interesting about energy from the Challenge, $18.7 \%$ thought "so and so", "a little" $10.3 \%$, while only $8.8 \%$ believed that they did not learn anything and $5.1 \%$ did not engage with it.

4.4.2 Educators questionnaire results. We handed out a post-activity questionnaire to 32 educators that were very actively involved in the project-related educational activities, as well as with the use of the Challenge by their students. The results from their answers to the questions related specifically to the Challenge can be seen in Fig. 11. To the question "Do you think the Challenge helped you save teaching time due to the introduction to sustainability and energy saving that it offered to students?" we see a quite positive result, with 40.6\% answering "Very much" and "Much", while $46.8 \%$ "So and so". We see an overwhelmingly positive response to the question "Do you think the Challenge meets the level of knowledge of your school's students?", with $87.5 \%$ answering "Very much" and "Much". Most importantly, to the question "'Have you noticed any change in your students' energy consumption behaviour after they played the game but before other pilot activities took place?", 65.6\% answered "Very much" and "Much". Interestingly, this number is not far off from the self-assessment of the students to a similar question (57.1\%, Table 2).

\section{DISCUSSION}

Regarding the results presented in the previous section, there are several interesting points that stand out. Players tended to complete a mission, after they started it, with very high probability. Apart from finding it engaging enough, this could also be interpreted as the Challenge meeting the knowledge level of the students; this also agrees with the educators' survey results in Fig. 11(b). The other thing that stands out is the different response in the 3 languages. This could be attributed on the one hand to the different age groups that utilized each language (Section 4.2); the Challenge was designed with smaller age groups in mind, so older students could be less interested in it. On the other hand, the group that was noticeably more engaged than the other two, were the Italian students that were also much more engaged with the competition aspects. Students that used English were in general the group that did not participate much, or at all, to the competition activities, while for Greek students were somewhere in between, with some schools participating and others not. Thus, it seems that competition was a major factor in motivating students to engage with the Challenge and carry on playing, combined with the fact that missions were engaging enough on their own.

Overall, the Challenge proved to be a successful component of the project toolset and the project's trials implementation strategy. The fact that we have had 3762 registered users by the project's end gives a clear indication of its success. An interesting result is the average session duration being above 14 minutes throughout the trials. This shows that the Challenge worked well, from an engagement level, given that missions are relatively short. Furthermore, the actual number among active players is considerably higher, because all users (i.e., even those we just registered and did not start a mission) were taken into account for this calculation. Moreover, the fact that students engaged with the platform outside of school hours, and even during weekends, is another good indicator that our design was effective in getting them interested in the Challenge. Finally, from our face-to-face interaction with teachers during pilots, they:

- Praised its overall design approach and simplicity.

- Noticed that the students did not have any major difficulties in using the Challenge, and also that it was very good that the students could use it outside of class hours, e.g., from their homes, due to lack of time to dedicate to this activity. In other words, they appreciated that students could play it without specific guidance.

- Praised its capacity as a short introduction to sustainability and energy, especially for students in primary schools.

- Stated that the capability to check what other schools were doing was a major engagement factor for students.

\subsection{Limitations}

A limitation of this work is that we did not conduct trials with study groups regarding the use of the Challenge. This was to a certain degree due to the fact that it was designed to serve as an introduction to sustainability and the project overall. Thus, student groups not using the Challenge or using it to a limited extent would change the overall design of the pilot activities. Moreover, among classes in the same school, in practice we saw that educators were 


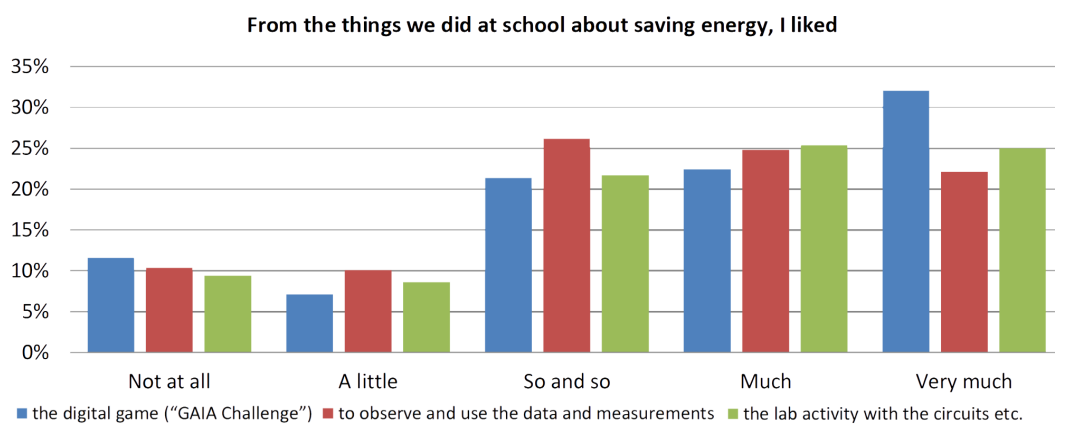

Figure 10: Students' responses to "from the things we did at school about saving energy, I liked" included in the post-activity questionnaire.

\begin{tabular}{l|l|l|l|l|l|l} 
& Not at all & A little & So and so & Much & Very much & I don't think I did this \\
\hline $\begin{array}{l}\text { "I learned a lot of interesting things about } \\
\text { energy by playing the Challenge" }\end{array}$ & $8.8 \%$ & $10,3 \%$ & $18,7 \%$ & $30,1 \%$ & $27,0 \%$ & $5,1 \%$
\end{tabular}

Table 2: Students' answers to the post-activity questionnaire.
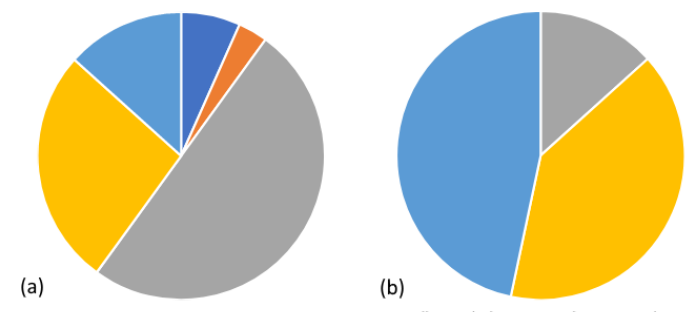

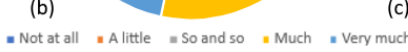

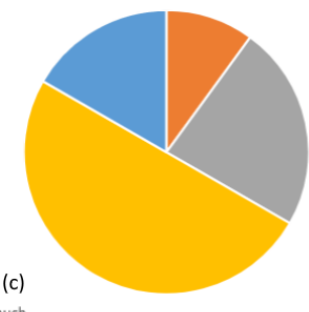

Figure 11: Responses from 32 educators that participated in the project to the following questions: (a) "Do you think the Challenge helped you save teaching time due to the introduction to sustainability and energy saving that it offered to students?", (b) "Do you think the Challenge meets the level of knowledge of your school's students?", (c) "Have you noticed any change in your students' energy consumption behaviour after they played the game but before other pilot activities took place?”

reluctant to divide students to separate groups, arguing they would prefer that all students followed the same work plan.

\section{CONCLUSIONS AND ONGOING WORK}

In this work, we presented our experiences from using a playful intervention in schools aiming for sustainability and energy awareness in the context of the GAIA project. The Challenge was based on a set of interactive quiz-based "missions" and utilized simple game mechanics, such as competition among groups, to actively engage players. By the end of the project, it had garnered 3762 registered users from students and educators in 3 countries. Our results indicate that to a great extent we achieved our goals; in terms of engagement, our data indicate significant students' engagement with the Challenge and that our competition game mechanic worked extremely well, while in terms of promoting sustainability, students reported increased awareness of related issues via post-activity surveys and educators reported changes in students' behavior. Our experience can be summarized as follows:

- Simple playful interventions can be very effective in school environments.
- Constraints in school environments should be taken into account.

- Game mechanics like competitions across classes/schools can work really well.

- You have to come up with a way to integrate the intervention to the everyday life of the school.

- Such an intervention is a good way to engage users when they cannot see the end of the journey from the start.

- The intervention should be part of a strategy that includes other tools.

\section{ACKNOWLEDGMENTS}

This work has been partially supported by the EU research project Green Awareness In Action, funded by the EC and the EASME under H2020 and contract number 696029. This document reflects only the authors' views and the EC and EASME are not responsible for any use that may be made of the information it contains.

\section{REFERENCES}

[1] 2015. Europe's buildings under the microscope: A country-by-country review of the energy performance of buildings. Technical Report. Buildings Performance 
Institute Europe (BPIE). ISBN: 9789491143014.

[2] 2015. Evans S., Global reaction: the Paris agreement on climate change. Carbon Brief Online Analysis. Available at https://www.carbonbrief.org/the-parisagreement-on-climate-change-the-world-reacts (Accessed $17 \mathrm{Feb} 2021$ ).

[3] 2020. Energy efficiency and behavioural change: Reducing carbon footprint through ICT and sustainable actions, https://cordis.europa.eu/article/id/421856energy-efficiency-and-behavioural-change.

[4] 2020. Energy performance of buildings directive (2018/844/EU) https:/ec.europa.eu/energy/topics/energy-efficiency/energy-efficientbuildings/energy-performance-buildings-directive_en.

[5] 2020. Games4Sustainability: Sustainability through serious games, https://games4sustainability.org.

[6] 2020. Green Awareness in Action, Horizon 2020 project. Available online http://gaia-project.eu.

[7] accessed online March 2021. Matomo Analytics, https://matomo.org/.

[8] (accessed online May 2021). Entropy project website, http://entropy-project.eu.

[9] accessed online May 2021. Game mechanics, Wikipedia, https://en.wikipedia.org/wiki/Game_mechanics.

[10] (accessed online May 2021). Greensoul, Persuasive Eco-awareness for User Engagement through Networked Data Devices, http://www.greensoul-h2020.eu/.

[11] accessed online May 2021. The GAIA Challenge, https://gaia-challenge.com/.

[12] (accessed online May 2021). Tribe H2020 project, http://tribe-h2020.eu/.

[13] Spartaco Albertarelli, Piero Fraternali, Sergio Herrera, Mark Melenhorst, Jasminko Novak, Chiara Pasini, Andrea-Emilio Rizzoli, and Cristina Rottondi. 2018 A Survey on the Design of Gamified Systems for Energy and Water Sustainability. Games 9, 3 (2018). https://doi.org/10.3390/g9030038

[14] Steven K Ayer, John I Messner, and Chimay J Anumba. 2016. Augmented reality gaming in sustainable design education. Fournal of Architectural Engineering 22, 1 (2016), 04015012.

[15] Jackie Barnes, Amy K Hoover, Borna Fatehi, Jesus Moreno-Leon, Gillian Smith, and Casper Harteveld. 2017. Exploring emerging design patterns in student-made climate change games. In Proceedings of the 12th International Conference on the Foundations of Digital Games. 1-6.

[16] Sebastian Deterding, Dan Dixon, Rilla Khaled, and Lennart Nacke. 2011. From Game Design Elements to Gamefulness: Defining Gamification. Proceedings of the 15th International Academic MindTrek Conference: Envisioning Future Media Environments, MindTrek 2011 11, 9-15. https://doi.org/10.1145/2181037.2181040

[17] Christo Dichev and Darina Dicheva. 2017. Gamifying education: what is known what is believed and what remains uncertain: a critical review. International Journal of Educational Technology in Higher Education 14 (12 2017). https: //doi.org/10.1186/s41239-017-0042-5

[18] Tawanna Dillahunt, Olga Lyra, Mary L Barreto, and Evangelos Karapanos. 2017 Reducing children's psychological distance from climate change via eco-feedback technologies. International fournal of Child-Computer Interaction 13 (2017), 1928.

[19] N. Dimitriou, A. Garbi, K. Vasilakis, A. Schoofs, A. Taha, M. Nikiforakis, S. Kotsilitis, T. G. Papaioannou, D. Kotsopoulos, C. Bardaki, F. Pursche, and N. Deliyski. 2018. ChArGED: Implementing a framework for improving energy efficiency in public buildings through IoTenabled energy disaggregation and serious games. In 2018 IEEE International Conference on Pervasive Computing and Communications Workshops (PerCom Workshops). 65-70.

[20] Alysson Diniz dos Santos, Francesco Strada, and Andrea Bottino. 2018. Approaching sustainability learning via digital serious games. IEEE Transactions on Learning Technologies 12, 3 (2018), 303-320.

[21] Michael Horn, Pryce Davis, Amartya Banerjee, and Reed Stevens. 2020. Fight the Power! Games, Thermostats, and the Energy Patriarchy. International fournal of Designs for Learning 11, 2 (2020), 118-129.

[22] Michael Stephen Horn, Amartya Banerjee, Pryce Davis, and Reed Stevens. 2016. Invasion of the Energy Monsters: A Spooky Game About Saving Energy. In Games+ Learning+ Society Conference, Vol. 12. 1851-1860.

[23] Robin Hunicke, Marc Leblanc, and Robert Zubek. 2004. MDA: A Formal Approach to Game Design and Game Research. AAAI Workshop - Technical Report 1 (01 2004).

[24] Daniel Johnson, Ella Horton, Rory Mulcahy, and Marcus Foth. 2017. Gamification and serious games within the domain of domestic energy consumption: A systematic review. Renewable and Sustainable Energy Reviews 73 (06 2017), 249-264. https://doi.org/10.1016/j.rser.2017.01.134

[25] Daniel Johnson, Ella Horton, Rory Mulcahy, and Marcus Foth. 2017. Gamification and serious games within the domain of domestic energy consumption: A systematic review. Renewable and Sustainable Energy Reviews 73 (06 2017), 249-264. https://doi.org/10.1016/j.rser.2017.01.134

[26] Erik Knol and Peter De Vries. 2011. EnerCities - A Serious Game to Stimulate Sustainability and Energy Conservation: Preliminary Results. eLearning Papers (07 2011)

[27] Alex Laskey and Ogi Kavazovic. 2011. OPOWER. XRDS 17, 4 (June 2011), 47-51. https://doi.org/10.1145/1961678.1961687
[28] Zdenko Mago. 2019. Increasing Awareness and Learning About Eco-Innovations Through Digital Games. In European Conference on Games Based Learning. Academic Conferences International Limited, 1013-1015.

[29] Alan Meier, Cecilia Aragon, Therese Peffer, Daniel Perry, and Marco Pritoni. 2011. Usability of residential thermostats: Preliminary investigations. Building and Environment 46, 10 (2011), 1891-1898.

[30] Luca Morganti, Federica Pallavicini, Elena Cadel, Antonio Candelieri, Francesco Archetti, and Fabrizia Mantovani. 2017. Gaming for Earth: Serious games and gamification to engage consumers in pro-environmental behaviours for energy efficiency. Energy Research \& Social Science 29 (2017), 95 - 102. https://doi.org/ 10.1016/j.erss.2017.05.001

[31] G. Mylonas, D. Amaxilatis, I. Chatzigiannakis, A. Anagnostopoulos, and F. Paganelli. 2018. Enabling Sustainability and Energy Awareness in Schools Based on IoT and Real-World Data. IEEE Pervasive Computing 17, 4 (Oct.-Dec. 2018), 53-63. https://doi.org/10.1109/MPRV.2018.2873855

[32] Georgios Mylonas, Dimitrios Amaxilatis, Lidia Pocero, Iraklis Markelis, Joerg Hofstaetter, and Pavlos Koulouris. 2019. An educational IoT lab kit and tools for energy awareness in European schools. International fournal of Child-Computer Interaction 20 (2019), 43 - 53. https://doi.org/10.1016/j.ijcci.2019.03.003

[33] Sofia Papavlasopoulou, Michail N. Giannakos, and Letizia Jaccheri. 2017. Empirical studies on the Maker Movement, a promising approach to learning: A literature review. Entertainment Computing 18 (2017), 57-78. https://doi.org/10. 1016/j.entcom.2016.09.002

[34] Filomena Pietrapertosa, Marco Tancredi, Monica Salvia, Monica Proto, Angelo Pepe, Michele Giordano, Nicola Afflitto, Giuliano Sarricchio, Senatro Di Leo, and Carmelina Cosmi. 2021. An educational awareness program to reduce energy consumption in schools. fournal of Cleaner Production 278 (2021), 123949. https://doi.org/10.1016/j.jclepro.2020.123949

[35] Gillian Puttick and Eli Tucker-Raymond. 2018. Building systems from scratch: An exploratory study of students learning about climate change. Fournal of Science Education and Technology 27, 4 (2018), 306-321.

[36] Michael Ro, Markus Brauer, Kathy Kuntz, Raj Shukla, and Ingo Bensch. 2017. Making Cool Choices for sustainability: Testing the effectiveness of a game-based approach to promoting pro-environmental behaviors. Journal of Environmental Psychology 53 (06 2017). https://doi.org/10.1016/j.jenvp.2017.06.007

[37] Michael Ro, Markus Brauer, Kathy Kuntz, Raj Shukla, and Ingo Bensch. 2017. Making Cool Choices for sustainability: Testing the effectiveness of a game-based approach to promoting pro-environmental behaviors. fournal of Environmental Psychology 53 (2017), 20 - 30. https://doi.org/10.1016/j.jenvp.2017.06.007

[38] Mara Soekarjo and H. Oostendorp. 2015. Measuring Effectiveness of Persuasive Games Using an Informative Control Condition. International fournal of Serious Games 2 (06 2015). https://doi.org/10.17083/ijsg.v2i2.74

[39] Giovanni Maria Troiano, Qinyu Chen, Ángela Vargas Alba, Gregorio Robles, Gillian Smith, Michael Cassidy, Eli Tucker-Raymond, Gillian Puttick, and Casper Harteveld. 2020. Exploring How Game Genre in Student-Designed Games Influences Computational Thinking Development. In Proceedings of the $2020 \mathrm{CHI}$ Conference on Human Factors in Computing Systems. 1-17.

[40] Femke van Horen, Arianne van der Wal, and Amir Grinstein. 2018. Green, Greener, Greenest: Can Competition Increase Sustainable Behavior? Journal of Environmental Psychology 59 (08 2018). https://doi.org/10.1016/j.jenvp.2018.08. 007

[41] Rob van Roy and Bieke Zaman. 2017. Why Gamification Fails in Education and How to Make It Successful: Introducing Nine Gamification Heuristics Based on SelfDetermination Theory. 485 - 509. https://doi.org/10.1007/978-3-319-51645-5_22

[42] Rob van Roy and Bieke Zaman. 2018. Need-supporting gamification in education: An assessment of motivational effects over time. Computers \& Education 127 (08 2018). https://doi.org/10.1016/j.compedu.2018.08.018

[43] Catherine Walker. 2017. Tomorrow's leaders and today's agents of change? Children, sustainability education and environmental governance. Children \& Society 31, 1 (2017), 72-83.

[44] Devon Wemyss, Roberta Castri, Francesca Cellina, Vanessa De Luca, Evelyn Lobsiger, and Vicente Carabias-Hutter. 2018. Examining community-level collaborative vs. competitive approaches to enhance household electricity-saving behavior. Energy Efficiency 11 (06 2018), 1-19. https://doi.org/10.1007/s12053-018-9691-z

[45] Dong Zhao, Andrew Mccoy, Jing Du, and Philip Agee. 2017. Interaction Effects of Building Technology and Resident Behavior on Energy Consumption in Residential Buildings. Energy and Buildings 134 (01 2017), 223-233. https://doi.org/10.1016/j.enbuild.2016.10.049 